\title{
A Remark with Regard to Inequalities for Some Sums
}

\section{Dragomir M. Simeunović}

Abstract. In this paper we consider some inequalities that are regarded to certain sums.

Inequalities that we shall use in this paper and that are proved in [1] are the following:

$$
b^{r}-a^{r} \geq r(b-a)(a b)^{\frac{r-1}{2}}, \quad b>a>0, r \geq 1
$$

and

$$
b^{s}-a^{s} \leq s(b-a)(a b)^{\frac{s-1}{2}}, \quad b>a>0,0<s \leq 1 .
$$

If in the inequality (1) we put

$$
b=\frac{1}{\left(n-\frac{1}{2}\right)^{2}+x^{2}-\frac{1}{4}}, \quad a=\frac{1}{\left(n+\frac{1}{2}\right)^{2}+x^{2}-\frac{1}{4}}, \quad x \neq 0,
$$

we shall get the inequality

$$
\begin{gathered}
\frac{1}{\left(\left(n-\frac{1}{2}\right)^{2}+x^{2}-\frac{1}{4}\right)^{r}}-\frac{1}{\left(\left(n+\frac{1}{2}\right)^{2}+x^{2}-\frac{1}{4}\right)^{r}} \\
\geq \frac{2 n r}{\left(\left(n^{2}+x^{2}\right)^{2}-n^{2}\right)^{\frac{r+1}{2}}}>\frac{2 n r}{\left(n^{2}+x^{2}\right)^{r+1}},
\end{gathered}
$$

where summing for $n=1,2, \ldots$, we get

$$
\sum_{n=1}^{\infty} \frac{2 n}{\left(n^{2}+x^{2}\right)^{2}}<\frac{1}{r\left(x^{2}\right)^{r}}, \quad r \geq 1, x \neq 0 .
$$

For $r=1$, from (4) we obtain

$$
\sum_{n=1}^{\infty} \frac{2 n}{\left(n^{2}+x^{2}\right)^{2}}<\frac{1}{x^{2}} .
$$

2010 Mathematics Subject Classification. Primary: 49A35, 90C30.

Key words and phrases. Inequalities for some sums. 
If in the inequality (2) we put

(6) $\quad b=\frac{1}{\left(n-\frac{1}{2}\right)^{2}+x^{2}+\frac{1}{4}}, \quad a=\frac{1}{\left(n+\frac{1}{2}\right)^{2}+x^{2}+\frac{1}{4}}, \quad x \neq 0$,

we get the inequality

$$
\begin{aligned}
& \frac{1}{\left(\left(n-\frac{1}{2}\right)^{2}+x^{2}+\frac{1}{4}\right)^{s}}-\frac{1}{\left(\left(n+\frac{1}{2}\right)^{2}+x^{2}+\frac{1}{4}\right)^{s}} \\
\leq & \frac{2 n s}{\left(\left(n^{2}+x^{2}+\frac{1}{2}\right)^{2}-n^{2}\right)^{\frac{s+1}{2}}}<\frac{2 n s}{\left(n^{2}+x^{2}\right)^{s+1}},
\end{aligned}
$$

as it is

$$
\frac{1}{\left(n^{2}+x^{2}+\frac{1}{2}\right)^{2}-n^{2}}<\frac{1}{\left(n^{2}+x^{2}\right)^{2}} .
$$

Summing for $n=1,2, \ldots$, from (7) we get

$$
\frac{1}{s\left(x^{2}+\frac{1}{2}\right)^{s}}<\sum_{n=1}^{\infty} \frac{2 n}{\left(n^{2}+x^{2}\right)^{s+1}}, 0<s \leq 1, x \neq 0 .
$$

For $s=1$, from (8) we obtain

$$
\frac{1}{x^{2}+\frac{1}{2}}<\sum_{n=1}^{\infty} \frac{2 n}{\left(n^{2}+x^{2}\right)^{2}}
$$

Inequalities (5) and (9) written in the form

$$
\frac{1}{x^{2}+\frac{1}{2}}<\sum_{n=1}^{\infty} \frac{2 n}{\left(n^{2}+x^{2}\right)^{2}}<\frac{1}{x^{2}}, \quad x \neq 0
$$

represent Mathieu's inequalities (see [2, p. 629]).

For $s=\frac{1}{r}, r \geq 1(0<s \leq 1)$, the inequality (8) reduces to

$$
\frac{r}{\left(x^{2}+\frac{1}{2}\right)^{\frac{1}{r}}}<\sum_{n=1}^{\infty} \frac{2 n}{\left(n^{2}+x^{2}\right)^{\frac{r+1}{r}}}, \quad r \geq 1 .
$$

From (4) and (11) we obtain inequality

$$
\frac{r}{\left(x^{2}+\frac{1}{2}\right)^{\frac{1}{r}}}<\sum_{n=1}^{\infty} \frac{2 n}{\left(n^{2}+x^{2}\right)^{\frac{r+1}{r}}}<\frac{1}{r\left(x^{2}\right)^{r}}, \quad r \geq 1, x \neq 0 .
$$

If in the inequality (1) we put

$$
b=\frac{1}{n-\frac{1}{2}+x^{2}}, \quad a=\frac{1}{n+\frac{1}{2}+x^{2}},
$$


for $r \geq 1$ and $x$ real number, we get inequality

$$
\begin{aligned}
& \frac{1}{\left(n-\frac{1}{2}+x^{2}\right)^{r}}-\frac{1}{\left(n+\frac{1}{2}+x^{2}\right)^{r}} \\
\geq & \frac{r}{\left(\left(n+x^{2}\right)^{2}-\frac{1}{4}\right)^{\frac{r+1}{2}}}>\frac{r}{\left(n+x^{2}\right)^{r+1}},
\end{aligned}
$$

where summing for $n=1,2, \ldots$, we get inequality

$$
\sum_{n=1}^{\infty} \frac{1}{\left(n+x^{2}\right)^{r+1}}<\frac{1}{r\left(x^{2}+\frac{1}{2}\right)^{r+1}}, \quad r \geq 1, x \text { is real number. }
$$

\section{REFERENCES}

[1] D.M. Simeunović, Sur certaines inégalités intégrales et leurs applications, Publ.. Inst. Math. 11 (25) (1971), 99-105.

[2] D.S. Mitrinović, J.E. Pečarić, A.M. Fink, Classical and New Inequalities in Analysis, Kluwer Academic Publishers, Dordrecht - Boston - London, 1993.

Dragomir M. Simeunović Mike Alasa 8

11000 BELGRADE

Serbia 